\title{
I-Cell Disease
}

\section{A HYPOTHESIS FOR THE STRUCTURE OF THE CARBOHYDRATE RECOGNITION SITE ON $\beta$-D- $N$-ACETYLHEXOSAMINIDASE}

\author{
By GEORGIRENE D. VLADUTIU \\ Division of Human Genetics, Department of Pediatrics at Children's Hospital, \\ State University of New York at Buffalo, Buffalo, NY 14222, U.S.A.
}

(Received 14 February 1978)

\begin{abstract}
I-cell disease (mucolipidosis II) is presented as a model for endo- and exo-cytosis phenomena in man. A hypothesis is presented for the structure of the carbohydrate recognition site on fibroblast-derived $\beta$-D- $N$-acetylhexosaminidase that may extend to the other affected hydrolases and that is responsible for specific uptake of the enzyme by fibroblasts. The proposed neuraminidase deficiency in I-cell disease is discussed in the light of its significance in influencing the final sugar sequence in the carbohydrate structure of the recognition site.
\end{abstract}

I-cell disease (mucolipidosis II) is a particularly interesting disorder because it provides a model for the exploration of basic endo- and exo-cytosis phenomena. Developments in the area of receptormediated endocytosis of lysosomal hydrolases and the potential impact of these studies on enzymereplacement therapy in lysosomal storage diseases increase the importance of the study of specific excretion and pinocytosis of lysosomal enzymes.

One of the main features of I-cell disease is that lysosomal enzymes are elevated in the medium of cultured fibroblasts from patients with the disease and are also elevated in the plasma of these patients. Hickman \& Neufeld (1972) showed that several hydrolases excreted by I-cell fibroblasts were altered in their ability to be pinocytosed by either non-I-cell or presumably I-cell-disease fibroblasts. Periodate oxidation experiments showed that specific pinocytosis of lysosomal enzymes by fibroblasts was dependent on a carbohydrate residue that acts as a recognition site on the enzymes and that this residue was altered in hydrolases excreted by I-cell fibroblasts (Hickman et al., 1974). Specifically, Kaplan et al. $(1977 a, b)$ have shown that a phosphomannosyl moiety is part of the recognition marker on human $\beta$-glucuronidase, $\beta$-hexosaminidase and $\beta$-galactosidase, which interacts with the fibroblast receptor. Sando \& Neufeld (1977) have also shown that phosphomannose is involved in the specific recognition and uptake of $\alpha$-iduronidase by fibroblasts.

Electrophoretic studies of fibroblast $\beta$-D- $N$-acetylhexosaminidase (EC 3.2.1.30) have shown that the intracellular isoenzyme pattern found in extracts of I-cell fibroblasts is more electropositive than the $\beta$-D- $N$-acetylhexosaminidase found in normal fibroblasts and the I-cell-fibroblast-excreted enzyme pattern is more electronegative than the normal fibroblast-excreted enzyme (Lie et al., 1973; Vladutiu \& Rattazzi, 1975). After neuraminidase treatment, the I-cell excreted $\beta$-D- $N$-acetylhexosaminidase electrophoretic pattern closely resembles the I-cell intracellular pattern, suggesting that the main difference between these two enzymes resides in their sialic acid content (Vladutiu \& Rattazzi, 1975). The mobilities of several other intracellular hydrolases in I-cell-disease fibroblasts have similar abnormalities (Champion \& Shows, 1977). Although $\beta$-D- $N$-acetylhexosaminidase will serve as the model enzyme here, it is generally believed that the alterations observed for this enzyme in I-cell-disease fibroblasts (hyperexcretion, hypersialylation, nonrecognition for uptake by deficient fibroblasts) are common to the other affected lysosomal enzymes in I-cell disease.

Several hypotheses for the defect in I-cell disease have been proposed. (1) An abnormal excretion of hydrolases occurs owing to 'leaky' lysosomes (Wiesmann et al., 1971). (2) Normally excreted acid hydrolases are defective and not recognized by fibroblasts for specific uptake (Hickman \& Neufeld, 1972). (3) Abnormal exocytosis occurs when primary lysosomes containing sialylated hydrolases fuse preferentially with the plasma membrane (Vladutiu \& Rattazzi, 1975). One explanation for the unusual isoenzyme forms of $\beta$-D- $N$-acetylhexosaminidase that are found in I-cell-fibroblast culture fluids is that, as a result of preferential exocytosis, the carbohydrate chain of the excreted enzyme is not completed normally. Therefore the specific carbohydrate residues necessary for recognition and uptake are never attached to the chain; rather, sialic acid is attached and the enzymes are directly ex- 
creted (Vladutiu \& Rattazzi, 1978). An alternative explanation is presented in the hypothesis proposed below.

The removal of the sialic acid on I-cell-fibroblastexcreted $\beta$-D- $N$-acetylhexosaminidase does not facilitate uptake of the enzyme (Vladutiu \& Rattazzi, 1978). Therefore it appears that, although galactose is obviously present, since sialic acid is usually attached to galactose, it is not the terminal recognition residue on the enzyme responsible for uptake by fibroblasts. This finding substantiates other reports (Hickman et al., 1974; Kaplan et al., 1977a).

The fact that sialic acid has no relevance in the impaired uptake of $\beta$-hexosaminidase by fibroblasts has made the significance of the reported neuraminidase deficiency in I-cell-disease fibroblasts (Thomas et al., 1976) and leucocytes (Strecker et al., $1976 b$ ) unclear. These considerations, combined with the recent finding of a phosphomannosyl recognition residue on certain hydrolases (Kaplan et al., 1977a,b; Sando \& Neufeld, 1977), have contributed to the development of the following hypothesis for the structure of the carbohydrate chain in $\beta$-D- $N$-acetylhexosaminidase derived from normal and I-cell fibroblasts and the effect that a neuraminidase deficiency may have in influencing this carbohydrate structure in I-cell disease.

\section{Hypothesis for the Structure of the Carbohydrate Recognition Chain of the Enzyme}

The residual $\beta$-D- $N$-acetylhexosaminidase found in extracts of I-cell fibroblasts may represent the same enzyme that is synthesized in the endoplasmic reticulum of normal fibroblasts complete with a normal oligosaccharide chain rather than the chain postulated (Vladutiu \& Rattazzi, 1975) This chain is probably not sialylated since the intracellular I-cell $\beta$-D- $N$-acetylhexosaminidase does not change mobility after neuraminidase treatment and electrophoretically resembles the neuraminidasetreated I-cell-excreted $\beta$-D- $N$-acetylhexosaminidase. Furthermore, this enzyme could not have been sialylated in the Golgi complex and then desialylated intracellularly if there is a deficiency in the appropriate neuraminidase isoenzyme. The core or side chain of carbohydrate residues ultimately involved in specific uptake could be in the following order in I-cell intracellular $\beta$-D- $N$-acetylhexosaminidase and linked to asparagine in the peptide portion: galactose- $\beta$-D- $N$-acetylglucosaminemannose-asparagine. The excreted I-cell $\beta$-D- $N$ acetylhexosaminidase could have this same structure with the exception that sialic acid has been attached to the galactose residue in the Golgi complex and becomes the terminal residue. The sialic acidgalactose $-N$-acetylglucosamine - mannose - aspara- gine sequence has been shown in other plasma glycoproteins (Kornfeld \& Kornfeld, 1976). Furthermore, these particular sugar residues are present in purified bovine $\beta$-glucuronidase (Himeno et al., 1974). In normal fibroblasts, this oligosaccharide chain would be desialylated in the primary lysosomes and then transfer to secondary lysosomes as the primary lysosomes fuse with phagocytic vacuoles. There the sugar residues would be sequentially removed until the shortened chain terminates in mannose. There is evidence that lysosomal hydrolases can act on intact glycoproteins rather than after protein degradation (Aronson \& de Duve, 1968). It is thus possible that in I-cell disease the reported neuraminidase deficiency could prevent initiation of the sequential removal of the carbohydrate residues on the sialylated $\beta$-D-Nacetylhexosaminidase in the primary lysosomes.

\section{Discussion}

It has been suggested that preferential fusion of primary lysosomes with the plasma membrane occurs to some extent in tissues from normal individuals, since plasma and seminal-fluid $\beta$-D- $N$-acetylhexosaminidases have electrophoretic properties similar to I-cell-excreted enzyme and neuraminidase treatment of all three enzymes also results in similar electrophoretic patterns (Vladutiu, 1976; Vladutiu \& Rattazzi, 1978). The hypothesis proposed in the present paper suggests that the primary lysosomes are the site of neuraminidase action in normal fibroblasts. Therefore this enzyme should be absent, defective or inhibited in I-cell fibroblasts. In normal cells, in which excretion is a constitutive function and which produce the $\beta$-D- $N$-acetylhexosaminidases found in plasma or seminal fluid, the neuraminidase may also be inhibited, absent or not accessible to the enzymes if they are preferentially excreted. The normal site for action of neuraminidase should be in the primary lysosomes, otherwise there is no feasible explanation for how sialylated enzyme is excreted from I-cell fibroblasts if not through direct fusion of primary lysosomes with the cell membrane.

The heterogeneity of lysosome populations within tissues and even within cells has been described, although it is not clear in many systems (Dean \& Barrett, 1976). Even the enzymic composition of lysosomes in certain cell types has been shown to be variable (Blum, 1976). It has been suggested that a whole series of presumably lysosomal acid neuraminidases may exist under separate genetic control in human fibroblasts (Kelly \& Graetz, 1977). Therefore it is possible that, in fibroblasts, different neuraminidase isoenzymes are active only at certain stages of lysosome development or the linkage of sialic acid to its carrier determines which neuraminidase isoenzyme will act upon it. The same 
restrictive criteria may govern the action or nonaction of other glycosidases.

Goldstone \& Koenig (1973) have suggested that, in rat kidney, lysosomal neuraminidase desialylates the acidic Golgi-derived lysosomal isoenzymes, resulting in the basic forms present in the secondary lysosomes. These workers neither proceed to postulate further degradation of the carbohydrate side chains of one glycosidase by another nor do they suggest that the degradation should stop with desialylation. It is difficult to explain, in the hypothesis proposed in the present paper, why sequential degradation of the oligosaccharide should terminate with mannose unless (a) mannose does not have to be terminal, but close to the terminus for recognition and uptake, (b) the mannose is not in a linkage or perhaps a configuration that is susceptible to mannosidase, especially if a phosphate group must be attached to the mannose for recognition, or $(c)$, if heterogeneity does exist in the hydrolase content of various lysosomes, perhaps mannosidase is not present or active at the time mannose is susceptible. Also mannose has been shown to be resistant to digestion in the sequential degradation by glycosidases of orosomucoid and fetuin (Aronson \& de Duve, 1968).

The possibility that a neuraminidase deficiency is the primary defect in I-cell disease has somehow been challenged by the finding of a neuraminidase deficiency in mucolipidosis I patients (Kelly \& Graetz, 1977; Strecker et al., 1976a; Cantz et al., 1977). It is noteworthy that there is an increase in endocellular sialic acid content in mucolipidosis I fibroblasts similar to mucolipodosis II. However, the hyperexcretion of lysosomal enzymes characteristic of mucolipidosis II fibroblasts is not found in mucolipidosis I fibroblasts (Kelly \& Graetz, 1977). Considering the biochemical differences between the two diseases, the respective neuraminidase deficiencies may in fact represent deficiencies of two different neuraminidase isoenzymes.

The $\beta$-D- $N$-acetylhexosaminidase excreted by normal fibroblasts is a higher-uptake form of enzyme than the enzyme found in normal fibroblast extracts (Kaplan et al., 1977b; Vladutiu \& Ratazzi, 1978). The excreted enzyme may contain more phosphomannosyl residues than the intracellular form or the residues on the excreted enzyme may contribute to the formation of a configuration more favourable for uptake. If there is an increase in phosphomannose or just phosphate groups on the excreted enzyme it may consist of just one or two residues, since the additional negative charges are not apparent in the electrophoretic mobility (Vladutiu \& Rattazzi, 1975) and only slightly apparent in isoelectric focusing (Kaplan et al., 1977b). Additions of only a few residues can have a major influence on uptake, in the light of Ashwell \& Morell's (1974) finding that one, two or three galactosyl residues on a circulating glycoprotein is adequate for recognition and uptake by liver cells.

Human platelet-derived $\beta$-D- $N$-acetylhexosaminidase is also a high-uptake enzyme (Kaplan et al., $1977 b$ ) and is probably enriched with phosphomannosyl residues. Human platelet membranes are known to possess at least two different mannosyltransferases and actively synthesize two prenyl phosphate mannoses (DeLuca, 1977). It is conceivable that $\beta$-D- $N$-acetylhexosaminidase from normal fibroblasts may acquire additional phosphomannosyl residues as it passes through the plasma membrane during fusion of secondary lysosomes with the membrane.

This hypothesis is novel in that it includes (a) a demonstration of how a primary deficiency in lysosomal neuraminidase may lead to an aberrant carbohydrate recognition residue in $\beta$-hexosaminidase that is probably common to all lysosomal enzymes in I-cell disease and $(b)$ a possible structural sequence for this residue in normal and I-cell-fibroblast-derived $\beta$-hexosaminidases.

I thank Dr. John B. Lloyd, Dr. Mario C. Rattazzi, and Dr. Patrick J. Carmody for their helpful discussions in the preparation of this manuscript.

\section{References}

Aronson, N. N., Jr. \& de Duve, C. (1968) J. Biol. Chem. 243, 4564-4573

Ashwell, G. \& Morell, A. G. (1974) Adv. Enzymol. Relat. Areas Mol. Biol. 41, 99-127

Blum, J. J. (1976) J. Cell. Physiol. 89, 457-472

Cantz, M., Gehler, J. \& Spranger, J. (1977) Biochem. Biophys. Res. Commun. 74, 732-738

Champion, M. J. \& Shows, T. B. (1977) Am. J. Hum. Genet. 29, 149-163

Dean, R. T. \& Barrett, A. J. (1976) Essays Biochem. 12, $1-40$

DeLuca, S. (1977) Biochim. Biophys. Acta 498, 341-348

Hickman, S. \& Neufeld, E. F. (1972) Biochem. Biophys. Res. Commun. 49, 992-999

Hickman, S., Shapiro, L. J. \& Neufeld, E. F. (1974) Biochem. Biophys. Res. Commun. 57, 55-61

Himeno, M., Hashiguchi, Y. \& Kato, K. (1974) J. Biochem. (Tokyo) 76, 1243-1252

Goldstone, A. \& Koenig, H. (1973) Biochem. J. 132, 267-282

Kaplan, A., Achord, D. T. \& Sly, W. S. (1977a) Proc. Natl. Acad. Sci. U.S.A. 74, 2026-2030

Kaplan, A., Achord, D. T. \& Sly, W. S. (1977b) J. Clin. Invest. 60, 1088-1093

Kelly, T. E. \& Graetz, G. (1977) Am. J. Med. Genet. 1, 31-46

Kornfeld, R. \& Kornfeld, S. (1976) Annu. Rev. Biochem. 45, 217-237

Lie, K. K., Thomas, G. H., Taylor, H. A. \& Sensenbrenner, J. A. (1973) Clin. Chim. Acta 45, 243-248

Sando, G. N. \& Neufeld, E. F. (1977) Cell 12, 619-627 
Strecker, G., Hondi-Assah, T., Fournet, B. \& Spik, G., Montreuil, J., Maroteaux, P., Durand, P. \& Farriaux. J. P. (1976a) Biochim. Biophys. Acta 444, 349-358

Strecker, G., Michalski, J. C., Montreuil, J. \& Farriaux, J. P. (1976b) Biomedicine 25, 238-240

Thomas, G. H., Tiller, G. E., Jr., Reynolds, T. W., Miller, C. S. \& Bace, J. W. (1976) Biochem. Biophys. Res. Commun. 71, 188-195
Vladutiu, G. D. (1976) N. Engl. J. Med. 295, 112-113

Vladutiu, G. D. \& Rattazzi, M. C. (1975) Biochem. Biophys. Res. Commun. 67, 956-964

Vladutiu, G. D. \& Rattazzi, M. C. (1978) Biochim. Biophys. Acta 539, 31-36

Wiesmann, U. N., Lightbody, J., Vassella, F. \& Herschkowitz, N. N. (1971) N. Engl. J. Med. 284, 109-110 